\title{
A LOCAL KERNEL PROPERTY OF CLOSED DERIVATIONS ON $C(I \times I)$
}

\author{
KATSUYOSHI NISHIO
}

\begin{abstract}
In this note we show a local behavior of closed derivations on $C([0,1] \times$ $[0,1])$, which is essentially different from one-dimensional derivations. Roughly speaking, any closed derivations on $C([0,1] \times[0,1])$ has a nonconstant kernel locally.
\end{abstract}

Our concerns are unbounded densely defined derivations on the Banach algebra $C(\Omega)$ of continuous real-valued functions on a compact Hausdorff space $\Omega$.

A (closed) derivation $\delta$ on $C(\Omega)$ means a (closed) linear map in $C(\Omega)$ defined on a dense subalgebra $\mathscr{D}(\delta)$ which satisfies the derivation property, i.e., $\delta(f g)=\delta(f) g+$ $f \delta(g)$ for every $f, g$ in $\mathscr{D}(\delta)$. Without loss of generality, we shall assume that the unit function 1 belongs to $\mathscr{D}(\delta)$ (so that $\delta(\mathbf{1})=0$ follows from the derivation property). The kernel and range of $\delta$ are denoted by ran $\delta$ and ker $\delta$, respectively.

A closed subset $E$ in $\Omega$ is called a self-determining set for $\delta$ if

$$
\left.\delta(f)\right|_{E}=0 \quad \text { whenever } f \in \mathscr{D}(\delta) \text { and }\left.f\right|_{E}=0 \text {, }
$$

where $\left.f\right|_{E}$ means the restriction of $f$ to $E$. If $E$ is a self-determining set, the formula $\delta_{E}\left(\left.f\right|_{E}\right)=\left.\delta(f)\right|_{E}$ defines a derivation $\delta_{E}$ with domain $\left\{\left.f\right|_{E} ; f \in \mathscr{D}(\delta)\right\}$. It is known (see [1, Lemma 4.1 or 2, Lemma 1.1.10]) that if $\delta$ is a closed derivation, for every open subset $U$ in $\Omega$ the closure $\bar{U}$ is a self-determining set for $\delta$ and, moreover, $\delta_{\bar{U}}$ is closable. For closable derivation $\boldsymbol{\delta}_{\bar{U}}$, the closure will be written by $\tilde{\delta}_{\bar{U}}$.

Closed derivations on the unit interval $I=[0,1]$ have been studied by various authors and recently the structure has been made clear (see references). On the contrary, even the derivations on $C(I \times I)$ are almost left untouched.

The following theorem gives a property of derivations on $C(I \times I)$ which clearly holds for partial derivatives, but which does not hold for derivations on $C(I)$.

THEOREM. For any closed derivation $\delta$ on $C(I \times I)$ with $\operatorname{ran} \delta=C(I \times I)$ and any open subset $V$ in $I \times I$, there is a nonempty connected open subset $U$ contained in $V$ such that $\operatorname{ker} \delta_{\bar{U}}$ contains some nonconstant functions in $\mathscr{D}\left(\delta_{\bar{U}}\right)$.

Proof. Without loss of generality we may assume that $V$ is connected. We consider two cases.

Case 1. There exists at least one nonempty open connected subset $W$ of $V$ such that $\delta_{\bar{W}}$ is not closed (but closable). In this case, it is easily shown that $\operatorname{ker} \tilde{\delta}_{\bar{W}}$

Received by the editors October 5, 1984 and, in revised form, January 14, 1985.

1980 Mathematics Subject Classification. Primary 46L05.

Key words and phrases. Derivation, closed operator, continuous function. 
contains a nonconstant function $F$. In fact, there is a function $G_{1}$ in $\mathscr{D}\left(\tilde{\delta}_{\bar{W}}\right)$, but not in $\mathscr{D}\left(\delta_{\bar{W}}\right)$. To such $G_{1}$ a function $G_{2}$ in $\mathscr{D}\left(\delta_{\bar{W}}\right)$ can be selected so as to satisfy $\delta_{\bar{W}} G_{2}=\tilde{\delta}_{\bar{W}} G_{1}$, because the assumption that $\operatorname{ran} \delta=C(I \times I)$ and the normality of the compact Hausdorff space $I \times I$ imply $\operatorname{ran} \delta_{\bar{W}}=C(\bar{W})$. Then the function $F=G_{1}-G_{2}$ is a desired function.

Therefore, there is a sequence of functions $\left\{f_{n}\right\}$ in $\mathscr{D}(\delta)$ such that

$$
\left.f_{n}\right|_{\bar{W}} \rightarrow F \quad \text { and }\left.\delta f_{n}\right|_{\bar{W}} \rightarrow 0 \quad(n \rightarrow \infty) .
$$

Let $U$ be any open connected subset in $W$ such that $\bar{U} \subset W$ and $\left.F\right|_{\bar{U}}$ is not constant. It is known (see [1, Lemma 2.3]) that there exists a function $h$ in $\mathscr{D}(\delta)$ such that $h=1$ on $\bar{U}$ and $\operatorname{supp} h \subset \bar{W}$. Then it is easily checked that

$$
h f_{n} \rightarrow f \quad \text { and } \delta\left(h f_{n}\right) \rightarrow g \quad(n \rightarrow \infty),
$$

where

$$
f=\left\{\begin{array}{ll}
h F & \text { on } \bar{W}, \\
0 & \text { on } \overline{W^{c}},
\end{array} \quad \text { and } g= \begin{cases}0 & \text { on } \bar{U}, \\
(\delta h) F & \text { on } \bar{W} \cap \bar{U}^{c}, \\
0 & \text { on } \bar{W}^{c} .\end{cases}\right.
$$

By closedness of $\delta, f$ belongs to $\mathscr{D}(\delta)$ and $\delta f=g$, so that $\left.f\right|_{\bar{U}}$ belongs to $\mathscr{D}\left(\delta_{\bar{U}}\right)$ and is not constant on $\bar{U}$, moreover, $\delta_{\bar{U}}\left(\left.f\right|_{\bar{U}}\right)=\left.\delta f\right|_{\bar{U}}=\left.g\right|_{\bar{U}}=0$.

Case 2 . The derivation $\delta_{\bar{U}}$ is always a closed derivation for every open connected subset $U$ of $V$. In particular, $\delta_{\bar{V}}$ is a closed derivation with $\operatorname{ran} \delta_{\bar{V}}=C(\bar{V})$. Hereafter $\delta_{\bar{V}}$ will be written by $\delta$ for the simplicity of notation.

Now suppose that our assertion is not true. Then for any open connected subset $U$ of $V$, ker $\delta_{\bar{U}}=\{\lambda \mathbf{1} ; \lambda$ real $\}$ holds. In particular, $\operatorname{ker} \delta=\{\lambda \mathbf{1} ; \lambda$ real $\}$. Take any point $x_{0}$ in $\bar{V}$, and let $\mathscr{D}_{0}=\left\{f \in \mathscr{D}(\delta) ; f\left(x_{0}\right)=0\right\}$. Then clearly $\delta$ is a closed linear one-to-one map from $\mathscr{D}_{0}$ onto $C(\bar{V})$, and $\delta^{-1}$ is a bounded linear operator on $C(\bar{V})$ by the closed graph theorem. Hence, for any fixed $x$ in $\bar{V}$, the map $C(V) \ni F \mapsto$ $\left(\delta^{-1} F\right)(x)$ is a bounded linear functional defined on $C(\bar{V})$, and by the Riesz representation theorem there exists a unique signed measure $\mu_{x}$ on $\bar{V}$ such that

$$
\left(\delta^{-1} F\right)(x)=\int_{\bar{V}} F d \mu_{x} \quad \text { for every } F \in C(\bar{V}) .
$$

Now take any connected open subset $U$ whose closure contains the points $x$ and $x_{0}$. Since the closed derivation $\delta_{\bar{U}}$ also satisfies that $\operatorname{ran} \delta_{\bar{U}}=C(\bar{U})$ and $\operatorname{ker} \delta_{\bar{U}}=$ $\{\lambda \mathbf{1} ; \lambda$ real $\}$, we can repeat the preceding argument with $\delta_{\bar{U}}$ instead of $\delta$, so that for any point $y$ in $\bar{U}$ there exists a unique signed measure $\mu_{y, \bar{U}}$ on $\bar{U}$ such that

$$
\left(\delta_{\bar{U}}\right)^{-1} F(y)=\int_{\bar{U}} F d \mu_{y, \bar{U}} \quad \text { for every } F \in C(\bar{U}) .
$$

This measure $\mu_{y, \bar{U}}$ can be considered as a measure on $\bar{V}$ by defining it to be zero outside $\bar{U}$. Then (2) can be written as

$$
\left.\left(\delta_{\bar{U}}\right)^{-1} F\right|_{\bar{U}}(y)=\int_{\bar{V}} F d \mu_{y, \bar{U}} \text { for every } F \in C(\bar{V}) \text { and } y \in \bar{U} .
$$


Here, if we put $f=\delta^{-1} F$, then $\delta f=F$ and $\delta_{\bar{U}}\left(\left.f\right|_{\bar{U}}\right)=\left.F\right|_{\bar{U}}$, and hence

$$
\left.\left(\delta_{\bar{U}}\right)^{-1} F\right|_{\bar{U}}(y)=f(y)=\left(\delta^{-1} F\right)(y)
$$

for any $y \in \bar{U}$. Accordingly, (1) and (2)' with $y=x$ imply

$$
\int_{\bar{V}} F d \mu_{x}=\int_{\bar{V}} F d \mu_{x, \bar{U}}
$$

for every $F \in C(\bar{V})$, from which follows $\mu_{x}=\mu_{x, \bar{U}}$. In particular, supp $\mu_{x}$ is contained in $\bar{U}$. Since $U$ can be selected arbitrarily so as to contain the points $x$ and $x_{0}, \mu_{x}$ must be an atomic measure with supp $\mu_{x} \subset\left\{x_{0}, x\right\}$ (cf. Remark following the proof). Therefore, (1) transforms into the form

$$
f(x)=\delta f\left(x_{0}\right) \mu_{x}\left(x_{0}\right)+\delta f(x) \mu_{x}(x) \text { for every } f \in \mathscr{D}_{0} .
$$

Since the value $\delta f\left(x_{0}\right)$ can be chosen independently for the values $f(x)$ and $\delta f(x)$, the point-mass $\mu_{x}\left(x_{0}\right)$ must be zero. Moreover, it can be easily shown that the point-mass $\mu_{x}(x)$ is also zero, by the derivation property. It follows that $\mathscr{D}_{0}$ is trivial, which leads to a contradiction. This concludes the proof.

REMARK. (1) The proof of Theorem remains valid in the case that the space $I \times I$ is replaced by a compact Hausdorff space $\Omega$ satisfying the following property:

For any three points $x, y$ and $z$ in $\Omega$, there is an open connected subset $U$ such that $\{x, y\} \subset U$ and $z \notin \bar{U}$.

Clearly the space $I$ does not possess the property.

(2) While the one-dimensional torus $T$ also satisfies (*), it is known (e.g. [6, Theorem 3.2.6]) that for any closed derivation on $C(T)$ the conclusion in the Theorem is invalid. Hence, it follows that the range of any closed derivation on $C(T)$ never coincides with $C(T)$.

We conclude with an example which is simple and relevant to our subjects.

EXAMPLE. The derivation on $C(I \times I)$ is defined as

$$
f(r, \theta)=\partial f(r, \theta) / \partial r
$$

for $f$ in $\mathscr{D}(\delta)$, where $(r, \theta)$ stand for the polar coordinates in $I \times I$. Every function $f$ in $\mathscr{D}(\delta)$ has every directional derivative at the origin, that is

$$
\lim _{\Delta r \rightarrow 0} \frac{f(\Delta r, \theta)-f(0)}{\Delta r}
$$

exists for every $\theta \in[0, \pi / 2]$, and all the values must coincide.

Then $\delta$ is certainly closed and $\operatorname{ran} \delta=C(I \times I)$. Moreover, it is easily seen that $\operatorname{ker} \delta=\{\lambda \mathbf{1} ; \lambda$ real $\}$ and ker $\delta_{\bar{U}}$ contains many nonconstant functions for every open subset $U$ whose closure does not contain the origin.

Acknowledgment. The author wishes to thank Professors Y. Haga and R. Nakamoto for a number of friendly and fruitful discussions. 


\section{REFERENCES}

1. C. J. K. Batty, Derivations on compact spaces, Proc. London Math. Soc. 42 (1981), 299-330.

2. F. M. Goodman, Closed derivations in commutative $C^{*}$-algebras, J. Funct. Anal. 39 (1980), 308-346.

3. H. Kurose, An example of a non-quasi well-behaved derivation in $C(I)$, J. Funct. Anal. 43 (1981), 193-201.

4. _ Closed derivation in C(I), Tôhoku Math. J. 35 (1983), 341-347.

5. $193-198$

6. J. Tomiyama, The theory of closed derivations in the algebra of continuous functions, Lecture Notes, Inst. Math., National Tsing Hua Univ., 1983.

Department of Applied Mathematics, Faculty of Engineering, Ibaraki University, Hitachi, IBARAKI 316, JAPAN 